between the alveoli and the mouth. Therefore, in EBC samples, not only volatiles, but also several other mediators with no volatile characteristics can be found and have been reported, including adenosine, different interleukins $(-4,-5$, -8), interferon- $\gamma$, etc. [3-5]. Regarding markers of lipid peroxidation, EBC contains isoprostanes and thiobarbituric acid-reactive substances [2].

The authors are right in saying that there are methodological limitations to this type of sampling. However, this is mainly due to the limited understanding of solute formation and dilution of samples, and the accuracy of some of the currently available methods for measuring mediators in EBC. I agree that ambient air may influence the levels of exhaled biomarkers in EBC and this is shown for hydrogen peroxide [6]. Volatility may be a problem when measuring mediators from $\mathrm{EBC}$, not because the sampling relies on this characteristic, but because if a molecule is volatile it is very hard to figure out the result of its equilibration between the gas and the fluid phase while breath condensation is ongoing. A good example is the ammonia measurement $[7,8]$.

Despite the misinterpretation of exhaled breath condensate, I believe that this review is a valuable source of knowledge and references on lipid peroxidation, with detailed information on the limitations and advantages of the current measuring methods.

\section{Horváth}

Dept of Pathophysiology, National Koranyi Institute for Pulmonology, Budapest, Hungary.

\section{References}

1. Wood LG, Gibson PG, Garg ML. Biomarkers of lipid peroxidation, airway inflammation and asthma. Eur Respir $J$ 2003; 21: 177-186.

2. Paredi P, Kharitonov SA, Barnes PJ. Analysis of expired air for oxidation products. Am J Respir Crit Care Med 2002; 166: S31-S37.

3. Huszar É, Vass G, Vizi É, et al. Adenosine in exhaled breath condensate in healthy volunteers and in patients with asthma. Eur Respir J 2002; 20: 1393-1398.

4. Shahid SK, Kharitonov SA, Wilson NM, Bush A, Barnes PJ. Increased interleukin- 4 and decreased interferon- $\gamma$ in exhaled breath condensate of children with asthma. Am J Respir Crit Care Med 2002; 165: 1290-1293.

5. Scheideler L, Manke HG, Schwulera U, Inacker O, Hammerle H. Detection of nonvolatile macromolecules in breath. A possible diagnostic tool? Am Rev Respir Dis 1993; 148: 778-784.

6. Latzin P, Griese M. Exhaled hydrogen peroxide, nitrite and nitric oxide in healthy children: decrease of hydrogen peroxide by atmospheric nitric oxide. Eur J Med Res 2002; 7: 353-358.

7. Effros RM, Hoaglang KW, Bosbous M, et al. Dilution of respiratory solutes in exhaled condensates. Am J Respir Crit Care Med 2002; 165: 663-669.

8. Vass G, Huszár É, Barát E, et al. Comparison of nasal and oral inhalation during exhaled breath condensate collection. Am J Respir Crit Care Med 2003; 167: 850-855.

From the authors:

We thank I. Horváth for his kind comments on our review "Biomarkers of lipid peroxidation, airway inflammation and asthma" [1], and agree that we have been imprecise with the use of the term "breath condensate". The paper would be improved by replacing this term with "exhaled breath" in relation to ethane, pentane and nitric oxide measurements.

While there is an intuitive explanation for the presence of volatile substances in exhaled breath, the mechanisms by which nonvolatile substances enter expired breath are poorly understood and need to be further investigated. 8-iso-prostaglandin $\mathrm{F}_{2 \alpha}$ and malondialdehyde have been measured in breath condensate as markers of lipid peroxidation [2]; however, it is, as yet, unknown whether this medium can be used for reliable measurement of antioxidant defences. Analysis of total and oxidised glutathione concentrations in induced sputum indicates that sputum supernatant is suitable for this purpose [3]. Hence, we stand by our conclusions in this area.

At the moment both sputum induction and breath condensate collection are promising techniques. The most useful sampling technique remains to be determined and this is an important area of future research. Comparison of both sampling methods in a head-to-head study is needed to resolve this issue.

\section{Wood*, P.G. Gibson*, M. Garg ${ }^{\#}$}

*Dept of Respiratory and Sleep Medicine, Hunter Medical Research Institute, John Hunter Hospital and \#Nutrition and Dietetics, University of Newcastle, New South Wales, Australia.

\section{References}

1. Wood LG, Gibson PG, Garg ML. Biomarkers of lipid peroxidation, airway inflammation and asthma. Eur Respir $J$ 2003; 21: 177-186.

2. Paredi P, Kharitonov SA, Barnes PJ. Analysis of expired air for oxidation products. Am J Respir Crit Care Med 2002; 166: S31-S37.

3. Dauletbaev N, Rickmann J, Viel K, Buhl R, Wagner TO, Bargon J. Glutathione in induced sputum of healthy individuals and patients with asthma. Thorax 2001; 56: 1318.

\title{
Noneosinophilic asthma
}

\section{To the Editors:}

We read with interest the article by BUIST [1] on similarities and differences between asthma and chronic obstructive pulmonary disease. We would like to make some comments on the nature of inflammation in asthma, which the author has mentioned to be predominantly eosinophilic. Patients have been noted to have severe asthma or suffer an exacerbation without an increase in the eosinophil population in the airways [2]. Based on several studies from 1995 onwards with data on eosinophil levels (cut-off values 2-4\%) on bronchial biopsy specimens, bronchoalveolar lavage fluid and sputum of asthmatic 